\title{
Rescheduling of Nodes Duty Cycles to Prevent Partitioning in Wireless Sensor Networks
}

\author{
M. MAHDAVI ${ }^{1, *}-$ M. ISMAIL ${ }^{2}$ \\ ${ }^{1}$ Department of Information Technology \& Computer Engineering, Payame Noor University (PNU), Iran \\ ${ }^{2}$ Department of Electrical, Electronic and System Engineering, Faculty of Engineering \& Built Environment, \\ UKM,Bangi, Selangor, Malaysia
}

\begin{abstract}
For the perfect operation of a WSN both requirements of sensing coverage and network connectivity are needed simultaneously. The method used in this work satisfies both of those requirements. For the sensing coverage, it uses randomized scheduling algorithm, in which each sensor node randomly selects one of the k predefined working subset. At any time only sensor nodes in one subset are active and nodes in the other subsets are in the sleep mode. Then, the algorithm turns on extra sensor nodes if necessary for the network connectivity. The problem of this method is some of extra-on nodes should be active more than one additional subset and even should be stay on all the time. Also extra-on nodes that are turned on to participate in other nodes routing, sometimes are subjected to many transmissions and receptions. So, they are faced with rapid battery depletion and this problem may lead to network partitioning. In this paper, we utilize rescheduling methods to minimize the number of extra on sensor nodes. By probing mechanism algorithms that allow for some nodes to change their working schedule, number of extra on nodes is reduced up to $35 \%$.
\end{abstract}

Keywords: wireless sensor networks, partitioning; sensing coverage, network connectivity, scheduling.

\section{INTRODUCTION}

Wireless Sensor networks (WSNs) consist of large number of tiny sensor nodes. Each sensor node includes sensing, data processing and communicating components. The sensor nodes will perform significant signal processing, computation, and network self-configuration to achieve scalable, robust, and long-lived networks [1]. More specifically, sensor nodes perform local processing to reduce communications and consequently, energy costs. Due to the small dimensions of sensor nodes (within several cube millimeters) [2], they have very limited power supplies. Moreover, because of the large number of sensors and/or hostile environments, charging or changing the batteries is impossible. Hence, energy efficiency is an important concern for WSNs to ensure extended period of operation using a limited battery power. Most of wireless sensor network applications use random deployment method to extend network life time. In random deployment method, the number of deployed sensor nodes is larger than an optimal deployment. If all the deployed sensor nodes simultaneously operate in the active mode, an excessive amount of energy would be wasted. In this case, if many sensors send packets, too much packet collisions will occur. A fundamental issue is to minimize the number of active nodes while still achieving an acceptable quality of service (sensing coverage) and network connectivity for the applications. Sensing coverage for sensing the area and detection of the events can be considered as the measure of quality of service for a sensor network [3]. The unit area is covered if any point in that area is within the sensing range of an active node. The network is connected if any active node can communicate with any other active node. With connectivity information collected by sensing coverage can be sent to the sink or base station.

Energy efficient connected coverage protocols consider sensing coverage and network connectivity requirements at the same time. Selecting a minimal set of active nodes in these protocols reduces power consumption and prolongs network lifetime. The works [4-10] have considered both problems of coverage and connectivity.

Liu et al. [9] have proposed joint scheduling method with considering requirements of sensing coverage and network connectivity. For the sensing coverage, randomized scheduling algorithm is employed that defines $k$ working subsets. At the time when sensor nodes are scattered in the area, each of them randomly joins one of the $k$ disjoint subsets. When the network is dense enough, each subset can cover the whole area. The $k$ disjoint subsets will work alternatively to extend network lifetime. At any time when sensor nodes in one subset are working, sensor nodes in the other subsets are in the sleep mode. To ensure network connectivity, firstly joint scheduling method starts to propagate the minimum hop count from the sink node. Each node after receiving a hop advertisement message puts the message in its buffer and defers the retransmission of the message after a back off time. At the end of this step, each node knows its minimum hop count to the sink node. Then, sensor nodes locally broadcasts their minimum hop count, their nodeID, their subset decision, the nodeIDs and subset decisions of their upstream nodes. The upstream nodes are the node's neighbors which their minimum hop counts are one less than the node's. The node neighbors with one more number of hop count than that of node are also called downstream of that node. Each sensor node records and maintains all the information it receives from its immediate neighbors. 
Secondly, sensor nodes will find at least a path to the sink node to create network connectivity. To minimize the number of broadcasts of working schedule updates, a sensor node updates its working schedule after it receives all the latest working schedules from its downstream nodes. Hence, this step should be started from nodes with maximum hop count that are located in the corner or of the area. In joint scheduling method, a node finds its path through its upstream nodes utilizing extra-on rule. When there is not upstream in the same subset with node, this rule allows for a randomly selected upstream to be active also in node's subset. Therefore, extra-on rule defines one or more additional working subset for some nodes. Those nodes are called extra-on nodes and they are under fast battery usage especially if they are active in all subsets. Extra-on nodes which working all the time are critical for the network connectivity because if they are turned off network will be partitioned.

Since extra-on nodes are turned on to participate in other nodes routing, they are at least subjected to one transmission and reception in addition to routing their packet itself. Even, some of those extra-on nodes are subjected to many transmissions and receptions and it will be added to the problem of fast battery depletion and probability of partitioning.

Therefore, reducing number of extra-on nodes is important to prevent partitioning. In this work, we introduce probing mechanism scheduling in joint scheduling method, which change some sensor nodes original working shift assigned by randomized scheduling algorithm. The operation of probing scheduling algorithms is explained in the next section.

\section{ALGORITHM DEVELOPMENT}

In the developed algorithm, randomized scheduling algorithm is fulfilled for the sensing coverage requirement. The steps of algorithm for the network connectivity can be summarized as following:

- Propagation of minimum hop count

- Probing mechanism scheduling

- Creating path to the sink node using extra-on rule

At the end of each step, information is broadcasted to the neighbors. After propagation of minimum hop count and exchange information with neighbors, probing mechanism scheduling is performed. Probing mechanism is a rescheduling process that changes some sensor nodes working shift which previously assigned by the randomized scheduling algorithm. Then, new working shift should be broadcasted to local neighbors. An example of nodes minimum hop counts in Fig. 1 is illustrated. In this figure, 100 nodes with a 40 meters communication range in the rectangular area are shown. The numbers indicate minimum hop count of each node from sink node.

Upstream nodes for nodes $A$ and $\mathrm{B}$, and downstream nodes for node $C$ have been highlighted in Fig. 2. Between two neighbors, the node with one less number of minimum hop count is called upstream node that is closer to the sink node. Node with one more number of minimum hop count is called downstream node. In this step, probing mechanism scheduling is employed.

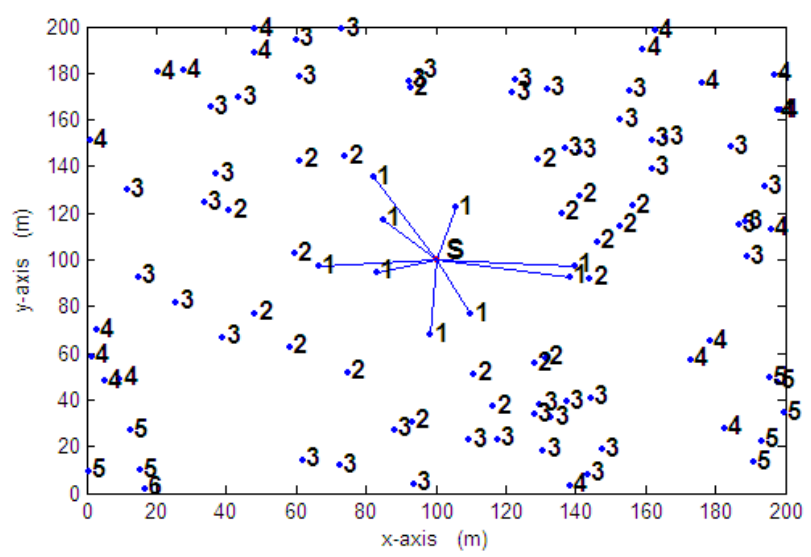

Fig. 1. Minimum hop counts from the Sink node $S$ 


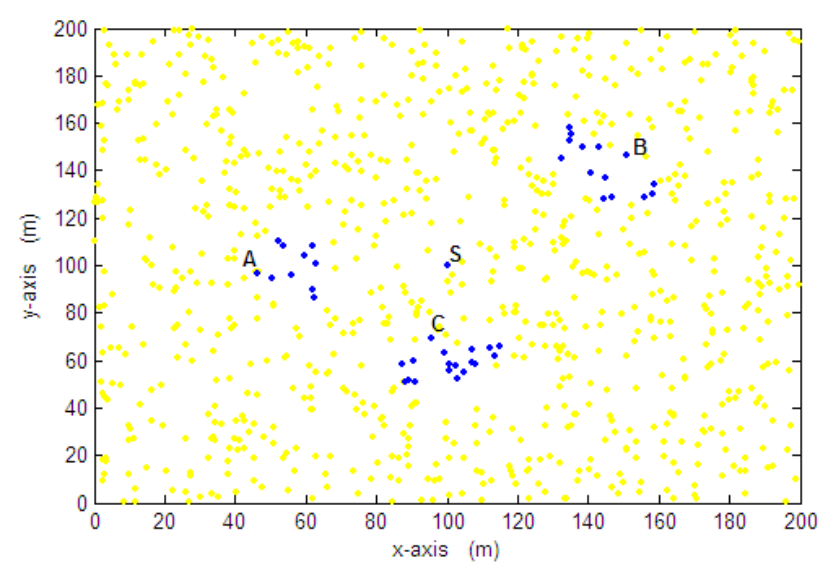

Fig. 2. Simulation scenario, upstream and downstream nodes

\subsection{Probing Mechanism scheduling}

After random deployment, each node has randomly joined one subset. To ensure network connectivity, each node needed to find a route to the sink through its upstream nodes. If a node could not find an upstream node working in its subset, one of its upstream nodes should work in extra subset that is an extra-on node. Hence, to reduce the number of extra-on nodes, upstream nodes of each node should work in node's subsets but it may be impossible in the case of nodes randomly joining subsets. In the probing mechanism step, each node probes its upstream nodes subsets and allow for an upstream node with more repeated subsets to change its working shift. Each node's subsets can only change once in this step. This step starts from nodes with maximum hop count values towards sink node.

Fig. 3 shows an example. Given $k$ equal 3, there are three subsets $k_{0}, k_{1}$ and $k_{2}$. Node $A$ works in subset $k_{0}$ and is searching routes toward sink node. To avoid creating extra-on nodes, one of the upstream nodes of $A$ should work in subset $k_{0}$. In the Fig. 3, nodes $B, C, D, E$ and $F$ are $A$ 's upstream nodes. Initially these node subsets are $k_{1}, k_{1}, k_{2}, k_{2}, k_{2}$ respectively. Maximum repeated subset is $k_{2}$, while any node doesn't work in subset $k_{0}$. Node $A$ selects one of the nodes $D, E$ or $F$ randomly and sends a message to it to change its scheduling subset from $k_{2}$ to $k_{0}$. The operation of probing mechanism1 (pbm1) for this example is summarized in Table 1.

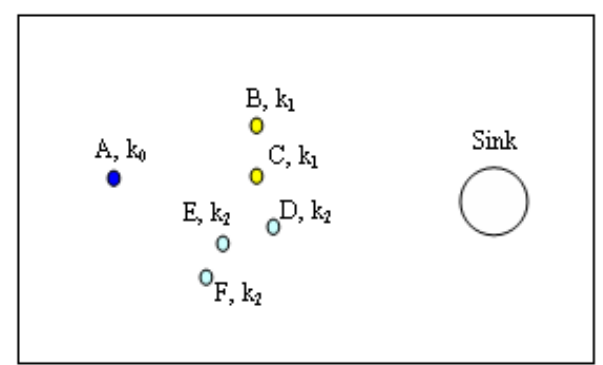

(a)

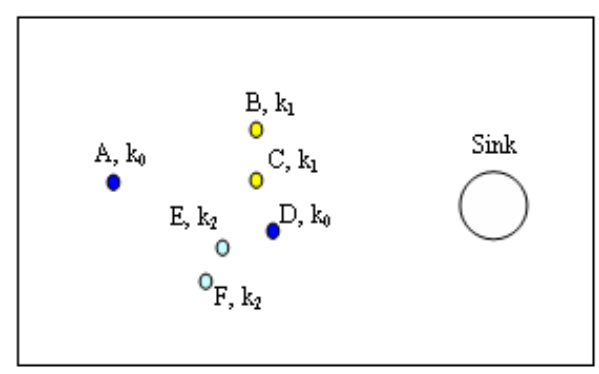

(b)

Fig. 3. (a) before pbm1 (b) after pbm1

Table 1. Operation for example of probing mechanism 1

\begin{tabular}{llllll}
\hline \multicolumn{1}{c}{ Parameter } & \multicolumn{5}{c}{ values } \\
\hline \hline Upstream nodes of $A$ & $B$ & $C$ & $D$ & $E$ & $F$ \\
Upstream nodes subsets & $k_{1}$ & $k_{1}$ & $k_{2}$ & $k_{2}$ & $k_{2}$ \\
Maximum repeated subset & $k_{2}$ & & & & \\
Node A's working subset & $k_{0}$ & & & & \\
$\begin{array}{l}\text { Upstream nodes subsets after } \\
\text { probing mechanism step }\end{array}$ & $k_{1}$ & $k_{1}$ & $k_{0}$ & $k_{2}$ & $k_{2}$ \\
\hline
\end{tabular}

At the end of this step, node $D$ working subset has been changed from $k_{2}$ to $k_{0}$ and node $A$ upstream nodes works in subsets $k_{0}, k_{l}, k_{2}$. In addition, updated working schedule is broadcasted to immediate neighboring sensor nodes. 
Although probing mechanism changes coverage scheduling, it should be performed after nodes know their minimum hop counts and neighboring information.

The above described algorithm, probing mechanism1 (pbm1) [11], has changed one more repeated subset to node's subset. $\mathrm{Pbm} 2$ changes one more repeated subset to a nonexistence subset. If there is no node working in subset $k_{x}$ among upstream nodes, one of the repeated subsets reschedules its working subset to $k_{x}$. In Fig. 4, no node works in subset $k_{1}$. After the probing mechanism, node $D$ works in subset $k_{l}$. Pbm3 algorithm probes upstream nodes. If there are repeated subsets, similar to pbm1, one more repeated subset changes to node's subset. Otherwise, if there are not repeated subsets, one of the upstream nodes subset assignment changes to node's subset (see Fig. 5). After probing mechanism, in the last step, each sensor node decides its extra working schedule and will find paths to the sink node through extra-on nodes.

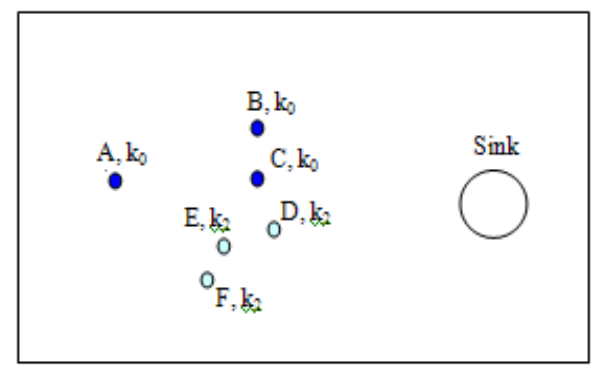

(a)

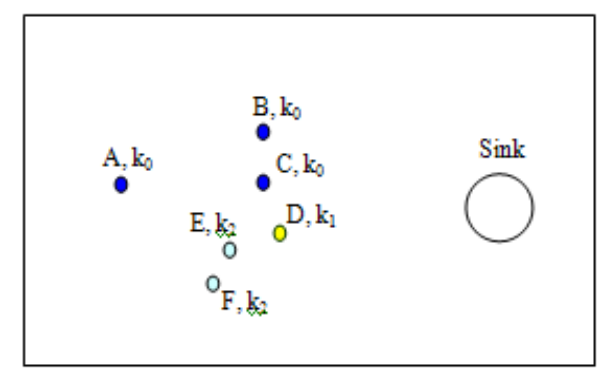

(b)

Fig. 4. (a) before pbm2 (b) after pbm2

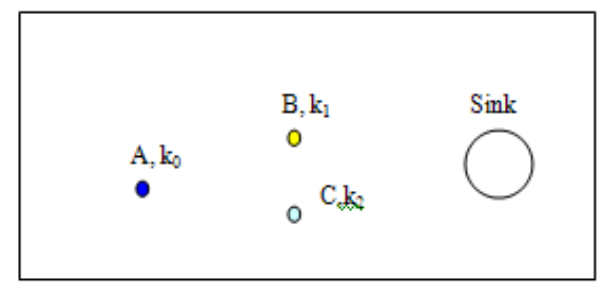

(a)

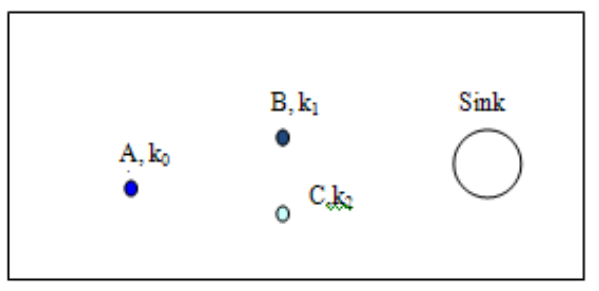

(b)

Fig. 5. (a) before pbm3 (b) after pbm3

\section{SIMULATION}

Generally, a large $k$ value means a larger number of subsets, and thus a subset may have to wait longer for its next turn to work. If the number of deployed sensor nodes is constant, a larger $k$ value means a smaller number of sensor nodes in each subset. This results in poorer network coverage. Proper $k$ or $n$ values are needed so that the energy can be saved with desirable network coverage. Hence, clear definition of the network coverage is needed. The relation between $n, k$ has been represented by [9] as following:

$$
n \geq\left[\frac{\ln (1-t)}{\ln \left(1-\frac{q}{k}\right)}\right]
$$

where $q=r / a, r$ is the size of the sensing area of each sensor, $a$ is the size of the whole field and $t$ is the network coverage intensity. The ideal value for the network coverage intensity is 1 . This ideal value implies that every point in the field is covered by at least one active sensor node at any given time with the probability of 1 . For the coverage intensity 0.9, $q=\pi / 400$ and $k=5$, the minimum number of sensor nodes needed for deployment is $n=1464$ from (1).

In this section a Matlab simulator has been implemented using CSMA/CA MAC layer protocol. Sensor nodes are deployed randomly in a 200 meters $\times 200$ meters region. The sink node is located at the center of the area. The sensing range of 10 meters is assigned for each sensor node. The total number of sensor nodes is selected according to (1) to meet any network coverage intensity. Because of very light traffic load, packet losses are mainly caused by network partition or channel errors. To prevent packet losses due to broadcast collision or channel errors, a perfect medium channel without medium contention is adopted. Under each simulation scenario, 100 runs with different random nodes have been executed.

In our previous study [12], we validated that the Packet Delivery Ratio (PDR) performance is in accordance with the work in [9]. PDR is defined as the ratio of total number of packets received at the sink node over the total number of transmitted packets from sensor nodes. Since the traffic load is very light, this metric can be an indicator of network 
connectivity. With the extra-on rule, network can always achieve 100 percent packet delivery ratio and so can achieve guaranteed network connectivity.

The ratio of extra-on nodes is defined as the ratio of the number of sensor nodes which remain active more than their scheduled subset to the total number of deployed sensor nodes. Ratio of extra-on sensor nodes for probing mechanism techniques for $k=3$ and network coverage intensity of 0.9 has been shown in Fig. 6. Maximum ratio of extra-on sensor nodes without probing scheduling is $9.84 \%$ for communication/sensing range of 2 . This ratio is reduced to $7.78 \%$ with probing mechanism1.

It can be seen that graph for pbm1 and pbm2 are almost same (With pbm2 maximum ratio was reduced to $7.83 \%$ with improvement of $20.42 \%$ ). Their differences are in their resulted topologies in each subset. For pbm3, ratio of extraon sensor nodes is $6.36 \%$ and reduction in the ratio of extra-on nodes is equal $35.37 \%$.

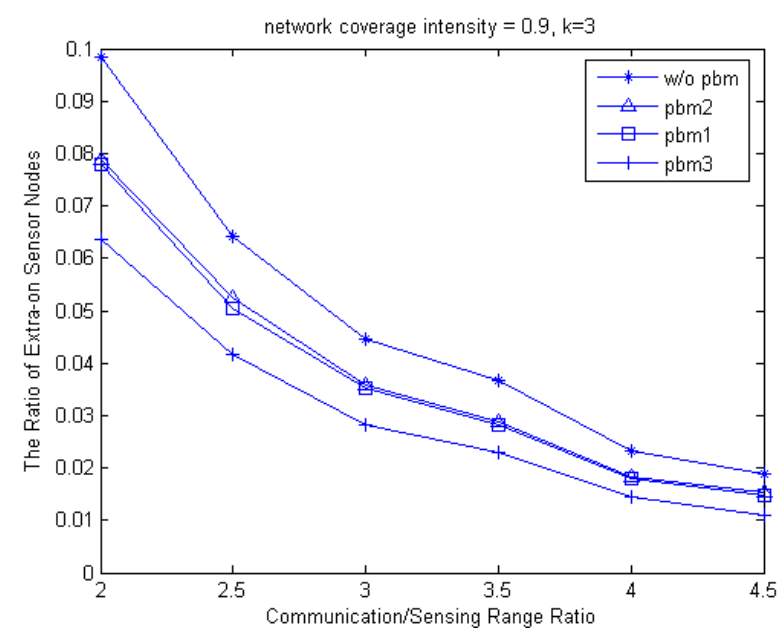

Fig. 6. Ratio of extra-on sensor nodes for pbm algorithms

\section{CONCLUSION}

In this paper we have considered two main requirements of wireless sensor networks consist of sensing coverage and network connectivity. Extra-on nodes have been turned on to create path to the sink node for some nodes but they were subjected to many transmissions/receptions. Also those extra-on nodes should be active in more than one subset and some of them worked all the time. Above mentioned problems discharged energy of those nodes rapidly. Since some of extra-on nodes are critical for network connectivity, their rapid battery depletion may lead to network partitioning. Probing mechanism has been implemented in joint scheduling method to reduce the number of extra-on nodes and to prevent partitioning. Using probing mechanism algorithms, if a node cannot find upstream node in its subset, one of its upstream nodes is allowed to change its working schedule based on probing mechanism rule. Simulation results have shown that the ratio of extra-on sensor nodes is reduced with the probing mechanism algorithms by $20.93 \%, 20.42 \%$ and $35.37 \%$.

\section{REFERENCES}

[1] Cerpa, A., Estrin, D. (2004). Ascent: Adaptive Self-Configuring Sensor Networks Topologies. IEEE Transactions on Mobile Computing, vol. 3, no. 2, p. 272-285.

[2] Warneke, B., Last, M., Leibowitz, B., Pister, K. (2001). Smart Dust: Communicating with a Cubic-Millimeter Computer. IEEE Journals \& Magazines, vol. 34, no. 1, p. 44-51.

[3] Yan, T., He, T., Stankovic, J. (2003). Differentiated Surveillance for Sensor Networks. Proceedings of the 1st international conference on Embedded networked, Nov. 2003.

[4] Tian, D., Georganas, D. (2005). Connectivity Maintenance and Coverage Preservation in Wireless Sensor Networks. Ad Hoc Networks J., vol. 3, no. 6, p. 744-761.

[5] Zhang, H., Hou, J. (2005). Maintaining Sensing Coverage and Connectivity in Large Sensor Networks. Ad Hoc \& Sensor Wireless Networks, vol. 1, no. 2, p. 89-124.

[6] Zhao, Q., Gurusamy, M. (2008).Lifetime Maximization for Connected Target Coverage in Wireless Sensor Networks. IEEE/ACM Transaction on Networking, vol. 16, no.6, p. 1378-1391.

[7] Zeng, Y., Sreenan, C. J., Xiong, N., Yang, L. T., Park, J. H. (2010).Connectivity and Coverage Maintenance in wireless Sensor Networks. The Journal of Supercomputing, vol. 52, no. 1, p. 23-46.

[8] Bai, X., Yun, Z., Xuan, D., Lai, T. H., Jia, W. (2010).Optimal Patterns for Four-Connectivity and Full Coverage in Wireless Sensor Networks. IEEE Transactions on Mobile Computing, vol. 9, no. 3, p. 435-448. 
[9] Liu, C., Wu, K., Xiao, Y., Sun, B. (2006). Random Coverage with Guaranteed Connectivity: Joint Scheduling for Wireless Sensor Networks. IEEE Transactions on parallel and distributed systems, vol. 17, no. 6, p. 562 - 575.

[10] Jameii, S. M., Faez, K., Dehghan, M. (2015). Multiobjective Optimization for Topology and Coverage Control in Wireless Sensor Networks. International Journal of Distributed Sensor Networks, vol. 2015, p. 1-11.

[11] Mahdavi, M., Ismail, M., Jumari K., Hanapi, Z. M. (2011). Probing Mechanism Scheduling for Connected Coverage Wireless Sensor Network. Information Technology Journal, vol. 10, no. 3, p. 579-584.

[12] Mahdavi, M., Ismail, M., Jumari, K., Hanapi, Z. M. (2009). Performance of a Connected Random Covered Energy Efficient Wireless Sensor Network. International Journal of Electrical, Computer, Energetic, Electronic and Communication Engineering, vol. 3, no. 5, p. 1141-1145. 